\title{
Management of spontaneous pneumothorax compared to British Thoracic Society (BTS) 2003 guidelines: a district general hospital audit
}

\section{*Andrew RL Medford, Justin CT Pepperell}

Department of Respiratory Medicine, Taunton and Somerset NHS Trust, Musgrove Park Hospital, Taunton, Somerset, TA1 5DA, UK

Received 15th February 2007; accepted 21st June 2007

\begin{abstract}
Background: In 1993, the British Thoracic Society (BTS) issued guidelines for the management of spontaneous pneumothorax (SP). These were refined in 2003.

Aim: To determine adherence to the 2003 BTS SP guidelines in a district general hospital.

Methods: An initial retrospective audit of 52 episodes of acute SP was performed. Subsequent intervention involved a junior doctor educational update on both the 2003 BTS guidelines and the initial audit results, and the setting up of an online guideline hyperlink. After the educational intervention a further prospective re-audit of 28 SP episodes was performed.

Results: Management of SP deviated considerably from the 2003 BTS guidelines in the initial audit - deviation rate $26.9 \%$. After the intervention, a number of clinical management deviations persisted (32.1\% deviation rate); these included failure to insert a chest drain despite unsuccessful aspiration, and attempting aspiration of symptomatic secondary SPs.

Conclusion: Specific tools to improve standards might include a pneumothorax proforma to improve record keeping and a pneumothorax care pathway to reduce management deviations compared to BTS guidelines. Successful change also requires identification of the total target audience for any educational intervention.

(c) 2007 General Practice Airways Group. All rights reserved.

ARL Medford and JCT Pepperell. Prim Care Resp J 2007; 16(5): 291-298.

doi:10.3132/pcrj.2007.00060
\end{abstract}

Keyw ords pneumothorax, BTS guidelines, audit, chest drain, pleural aspiration

\section{Introduction}

Pneumothorax is an important differential diagnosis to be considered in any of the substantial number of patients presenting in primary care with acute or sub-acute dyspnoea with or without chest pain. However, the absolute number of cases is low. In a recent epidemiological study using the General Practice Research Database and Hospital Episode Statistics data, 9.8 consultations per 100,000 women per year, and 24.0 consultations per 100,000 men per year, occurred because of primary or secondary pneumothorax. ${ }^{1}$ Mortality data reveal a mortality rate of 0.62 per million/year and 1.26 per million/year for women and men, respectively, for primary and secondary pneumothorax. Mortality is higher in patients aged over 55 years. Primary spontaneous pneumothorax (PSP), without underlying lung disease, is rarely fatal but has significant economic impact. Secondary spontaneous pneumothorax (SSP), in patients with underlying lung disease, is more serious.

In 1993, the British Thoracic Society (BTS) issued spontaneous pneumothorax (SP) management guidelines. ${ }^{2}$ The algorithm recommended observation alone in selected patients, or aspiration as initial intervention in all SP cases where observation was insufficient, irrespective of pneumothorax size, underlying lung disease or number of previous episodes.

The 1993 BTS guidelines were refined in 2003 to clarify several issues including: pneumothorax size estimation; initial procedure in both PSP and SSP; treatment of SSP; and specialist referral timing. ${ }^{3}$ The major changes were:

\footnotetext{
* Corresponding author: Department of Respiratory Medicine, Chest Clinic, Level 6, Derriford Hospital, Plymouth, Devon, PL6 8DH, UK Tel: +44 (0)1752 763869 Fax: +44 (0)1752 763864 E-mail: andrewmedford@hotmail.com
} 
1. Use of the $2 \mathrm{~cm}$ threshold (a $49 \%$ pneumothorax assuming a $10 \mathrm{~cm}$ hemithorax) for intervention. Most SP sizes were underestimated in the 1993 system because of the volume effect. ${ }^{4}$

2. Aspiration for PSP $>2 \mathrm{~cm}$, or observation if $<2 \mathrm{~cm}$ and minimal symptoms.

3. Insertion of chest drain for SSP $>2 \mathrm{~cm}$ or with significant symptoms, with aspiration if minimal symptoms and $<2 \mathrm{~cm}$.

4. Referral to a respiratory physician at 48 hours if patient fails to respond to treatment.

5. Referral to a thoracic surgeon at 3-5 days (3 for SSP) if persistent air leak or failed re-expansion.

The 2003 BTS chest drain guidelines were developed to reduce complications by teaching systematic methods of insertion. ${ }^{5}$

There is one other international SP guideline - from the American Thoracic Society (ATS) ${ }^{6}$ - which has broad similarities with the BTS guideline. Both are based on an assessment of pneumothorax size and the presence of symptoms, and both have similar recommendations for the timing of the surgical consultation and hospitalisation. There are two important differences, however; the ATS guideline does not use the $2 \mathrm{~cm}$ rule for size estimation, nor does it advocate the use of pleural aspiration.

In general, the majority of patients with SP will be managed in the Emergency Room (ER) or Accident and Emergency (A\&E) department, although general practitioner (GP) referrals may also be managed in acute medical assessment units.

The aim of this study, therefore, was to perform an initial retrospective audit of SP management according to 2003 BTS guideline criteria, and then to perform a prospective re-audit following an intervention which involved a detailed educational meeting for all junior doctors in the medical directorate and provision of an online hyperlink to the 2003 guidelines.

\section{Methods}

\section{Initial case notes retrospective audit}

Patients seen at Musgrove Park Hospital between January 2004 and January 2005 with acute SP were identified from ward discharges with the diagnostic code for "spontaneous pneumothorax". Relevant case notes and digital chest radiographs were retrieved. Cases were rejected if not located after two searches, or if the diagnosis was not SP.

Audit criteria were assessed as follows:

1. Documentation of age, gender, smoking status, occupation and source of referral

2. Correct diagnosis of PSP or SSP

3. Correct CXR interpretation using revised BTS algorithm for assessment

4. Correct management based on revised BTS algorithm
5. Initial intervention:

a. if observation, recorded use of oxygen

b. if aspiration, recorded use of "safe zone", aspiration volume, operator rank

c. if chest drain, recorded use of "safe zone", and anaesthesia (as per BTS chest drain guidelines), operator rank

6. Timing of specialty referral (BTS guidelines)

7. Follow-up arrangements and timing (BTS guidelines)

8. Advice on smoking cessation (BTS guidelines)

\section{Intervention}

Having identified problems in the initial audit, a detailed educational update on the revised BTS guidelines and the initial audit findings was presented to all Trust medical junior doctors. This consisted of a 60-minute teaching session given by a respiratory consultant and specialist registrar in bleepprotected time which focussed on the initial audit results, the 2003 changes to the BTS guidelines, and practical points concerning pleural procedures and when to contact the respiratory team. Post-study analysis revealed that $66.7 \%$ of doctors involved in the post-intervention group attended this education update, and that $67.3 \%$ of doctors from the original retrospective audit were from the same directorate i.e. both audits were matched for doctor specialty. An online hyperlink to the updated BTS guidelines on the intranet was generated and was accessible at all terminals.

\section{Prospective re-audit}

Following the educational intervention, a prospective re-audit of 28 further consecutive episodes of acute SP was undertaken.

\section{Statistical analysis}

Statistical analysis was performed using GraphPad Prism 4 software using Fishers exact test or Chi Squared test for contingency table analysis. Non-contingency table data were found to be normally distributed (by Ryan-Joiner analysis) and were analysed by unpaired t-test. $P$ values less than 0.05 were deemed significant. Contingency data are recorded in percentages with odds ratios, 95\% confidence intervals and $p$ values. Non-contingency data are recorded with mean values, standard errors and $p$ values.

\section{Results Patients}

Fifty-two SP episodes occurred in 52 patients in the initial audit, and 28 episodes occurred in 28 patients in the prospective re-audit. Five cases were excluded in total: three SP-coded cases were excluded because the patients' case notes could not be located after two searches, and two cases were excluded because they did not have SP coding and were actually identified as traumatic pneumothorax. The proportion of PSPs and SSPs with any underlying lung disorders, their age, gender, smoking status, and recording of 
Table 1. Proportion of pneumothoraces, breakdown of underlying lung disease as well as age, gender, smoking status and occupation for audit and re-audit.

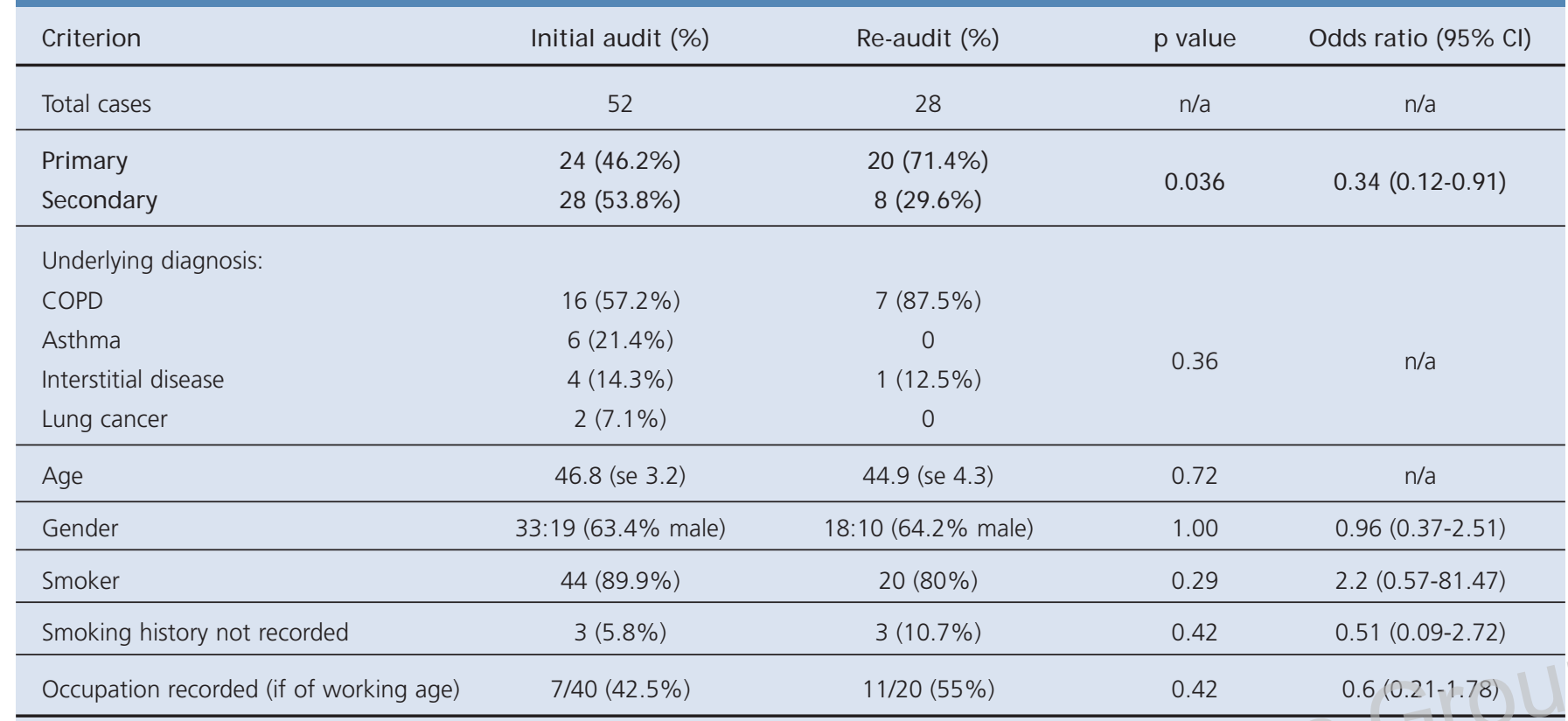

PSP, primary spontaneous pneumothorax; SSP, secondary spontaneous pneumothorax; se, standard error; $95 \% \mathrm{Cl}$, 95\% confidence intervals for odds ratios.

\section{Table 2. Referral sources and proportion of chest racilograph errors for both cohorts.}

\begin{tabular}{|c|c|c|c|c|}
\hline Criterion & initial audit (\%) & Re-audit (\%) & $p$ value & Odds ratio $(95 \% \mathrm{Cl})$ \\
\hline Total cases & 52 & 28 & $\mathrm{n} / \mathrm{a}$ & $\mathrm{n} / \mathrm{a}$ \\
\hline$A \& E$ & $29(55.8 \%)$ & $20(71.4 \%)$ & \multirow{3}{*}{0.34} & \multirow{3}{*}{ n/a } \\
\hline GP & $20(38.4 \%)$ & $6(21.4 \%)$ & & \\
\hline Radiology dept & $1(1.9 \%)$ & 0 & & \\
\hline CXR error & $9(17.3 \%)$ & $5(17.9 \%)$ & 1.00 & $0.96(0.28-3.21)$ \\
\hline \multicolumn{5}{|l|}{ CXR error: } \\
\hline No comment at all & $6(11.5 \%)$ & $5(17.9 \%)$ & 0.34 & $\mathrm{n} / \mathrm{a}$ \\
\hline
\end{tabular}

95\% Cl, as for Table 1; A\&E, accident and emergency; GP, general practice; CXR, chest radiograph.

occupation and smoking status, are displayed in Table 1.

The cohorts were well matched for age and gender, with a male preponderance and expected bimodal age distribution. There was an increase in proportion of PSP cases in the reaudit $(71.4 \%$ v $46.2 \%$, OR $0.34(0.12-0.91), p=0.036)$.

The referral sources and composition of chest radiograph (CXR) errors are displayed in Table 2. There was no overall difference in referral sources in the cohorts, with A\&E being the commonest referral pathway. It should be noted that $27 \%$ (initial audit) and 26\% (re-audit) of the A\&E referrals had previously been seen by their GP but were managed in A\&E. In addition, all of the GP referrals were referred directly to the admitting medical team. The proportion of CXR errors was similar in both audits but there were no failures to record 
Table 3. Frequency and composition of management errors for both cohorts, initial and subsequent interventions Documentation of pleural aspiration and chest drain insertion methods.

\begin{tabular}{|c|c|c|c|c|}
\hline Criterion & Initial audit (\%) & Re-audit (\%) & $p$ value & Odds ratio $(95 \% \mathrm{Cl})$ \\
\hline Total cases & 52 & 28 & $\mathrm{n} / \mathrm{a}$ & $\mathrm{n} / \mathrm{a}$ \\
\hline Incorrect management: & $14(26.9 \%)$ & $9(32.1 \%)$ & 0.29 & $0.56(0.21-1.52)$ \\
\hline Aspiration, not ICD & $4(7.7 \%)$ & $3(10.7 \%)$ & & \\
\hline ICD, not aspiration & $5(9.6 \%)$ & $2(7.1 \%)$ & 0.37 & $\mathrm{n} / \mathrm{a}$ \\
\hline Observation (no oxygen) & $5(9.6 \%)$ & $2(7.1 \%)$ & & \\
\hline No ICD after failed aspiration & 0 & $2(7.1 \%)$ & & \\
\hline \multicolumn{5}{|l|}{ Initial intervention: } \\
\hline ICD & $35(67.3 \%)$ & $9(32.1 \%)$ & 0.004 & $4.34(1.63-11.6)$ \\
\hline Aspiration & $12(23.1 \%)$ & $10(35.7 \%)$ & 0.29 & $0.54(0.19-1.48)$ \\
\hline Observation & $5(9.6 \%)$ & $9(32.1 \%)$ & 0.032 & $0.22(0.067-0.76)$ \\
\hline \multicolumn{5}{|l|}{ Subsequent intervention: } \\
\hline ICD alone & $43(82.3 \%)$ & $13(46.4 \%)$ & 0.002 & $5.51(1.96-15.5)$ \\
\hline ICD and suction & $14(26.9 \%)$ & $3(10.7 \%)$ & 0.15 & $3.07(0.79-11.8)$ \\
\hline \multirow{2}{*}{$\begin{array}{l}\text { Aspiration } \\
I C D\end{array}$} & 12 & 10 & \multirow{3}{*}{$n / a$} & \multirow{2}{*}{3} \\
\hline & 43 & 13 & & \\
\hline \multirow{3}{*}{$\begin{array}{l}\text { Aspiration position recorded } \\
\text { Aspiration volume not recorded } \\
\text { Mean aspiration volume (litres) }\end{array}$} & $5(41.7 \%)$ & $8(80 \%)$ & & \multirow{3}{*}{$\begin{array}{c}0.18(0.026-1.23) \\
15.4(0.73-323.1) \\
n / a\end{array}$} \\
\hline & $5(41.7 \%)$ & 0 & 0.04 & \\
\hline & $1.78($ se 0.34$)$ & 1.27 (se 0.22 ) & 0.21 & \\
\hline \multirow{4}{*}{$\begin{array}{l}\text { ICD position recorded } \\
\text { Mean ICD gauge (Fr) } \\
\text { ICD gauge not recorded } \\
\text { Lignocaine recorded }\end{array}$} & $19(40.4 \%)$ & $11(84.6 \%)$ & 0.01 & \multirow{2}{*}{$\begin{array}{c}0.14(0.028-0.75) \\
n / a\end{array}$} \\
\hline & $19.0($ se 1.46$)$ & 20.4 (se 2.38 ) & 0.62 & \\
\hline & $15(34.9 \%)$ & $3(23.1 \%)$ & 0.51 & $1.79(0.43-7.49)$ \\
\hline & $19(44.2 \%)$ & $4(30.8 \%)$ & 0.52 & $1.78(0.47-6.69)$ \\
\hline
\end{tabular}

$95 \% \mathrm{Cl}$, as for Table 1; ICD, intercostal chest drain; Fr, French.

the size of pneumothorax in the re-audit; all errors were due to failure to document any CXR findings. In the re-audit, there was a significant improvement in use of the revised BTS 2003 algorithm for assessing the pneumothorax size $(32.1 \% \mathrm{v}$ $2.9 \%$, OR 0.041 (0.004 - 0.34), $\mathrm{p}=0.0002)$.

The frequency and composition of management deviations from the revised BTS guidelines are displayed in Table 3. There was no change in proportion of deviations post-intervention (32.1\% v 26.9\%, OR 0.56 (0.21 - 1.52), $\mathrm{p}=0.29)$. Assuming an "ideal" $100 \%$ standard according to the BTS guidelines, both the initial audit (OR $0.013(0.0008$ $-0.23), p<0.0001)$ and re-audit (OR $0.01(0.0006-0.18)$, $\mathrm{p}<0.0001)$ fell significantly below BTS standards. Moreover, this persisted even if the standard for the audit criteria was dropped to a $90 \%$ agreement rate.

Three types of error were noted in both audits: the inappropriate use of aspiration for SSPs $>2 \mathrm{~cm}$ instead of chest drain; the inappropriate use of chest drain instead of aspiration for PSPs without tension; and the failure to use oxygen with observation overnight. In the re-audit, a fourth error was identified - failure to proceed to chest drain insertion when aspiration was unsuccessful.

The initial and subsequent interventions are summarised in Table 3. In the re-audit, there was a significant reduction in chest drain insertion as both the initial $(32.1 \% \vee 67.3 \%$, OR $4.34(1.63$ - 11.6), $p=0.0004)$ and subsequent $(46.4 \% \vee$ $82.3 \%$, OR $5.51(1.96-15.5), p=0.0002)$ intervention, as well as a significant increase in 'observation only' as the initial intervention ( $32.1 \% \vee 9.6 \%$, OR $0.22(0.067-0.76), p=0.032)$.

Further details on recording aspiration and chest drain procedures are summarised in Table 3. In all cases, Seldinger type drains (Cook, Portex) were used with either moderate (1624 French (Fr) Gauge) to large (28-36Fr), or small (10-14Fr), gauge. A significant proportion of chest aspiration procedures 
Table 4. Operator rank for pleural procedures, referral rates to specialist services and response times, follow up and advice.

\begin{tabular}{|c|c|c|c|c|}
\hline Criterion & Initial audit (\%) & Re-audit (\%) & $p$ value & Odds ratio $(95 \% \mathrm{Cl})$ \\
\hline Total cases & 52 & 28 & $\mathrm{n} / \mathrm{a}$ & $\mathrm{n} / \mathrm{a}$ \\
\hline \multicolumn{5}{|l|}{ Operator ranks: } \\
\hline $\mathrm{SHO}$ & $37(71.1 \%)$ & $15(79.0 \%)$ & \multirow{3}{*}{0.12} & \multirow{3}{*}{$\mathrm{n} / \mathrm{a}$} \\
\hline SpR & $14(26.9 \%)$ & $2(10.5 \%)$ & & \\
\hline Cons & $1(1.9 \%)$ & $2(10.5 \%)$ & & \\
\hline \multicolumn{5}{|l|}{ Referrals: } \\
\hline No referral to respiratory & $5(9.6 \%)$ & $8(28.6 \%)$ & 0.05 & $0.27(0.07-0.91)$ \\
\hline Time to resp review (days) & 1.05 (se 0.09) & $0.81(0.14)$ & 0.16 & $\mathrm{n} / \mathrm{a}$ \\
\hline Resp review $>48$ hrs & $5(9.6 \%)$ & $1(3.6 \%)$ & 0.66 & $2.87(0.32-25.9)$ \\
\hline Thoracic surgical referral & $18(34.6 \%)$ & $5(17.8 \%)$ & 0.13 & $2.44(0.79-7.49)$ \\
\hline Time to surg review (days) & 4.1 (se 0.65) & 4.7 (se 3.67) & 0.79 & $n / a$ \\
\hline Surg review $>72$ hrs & $5(9.6 \%)$ & $3(10.7 \%)$ & 0.71 & $0.78(0.17-3.56)$ \\
\hline \multicolumn{5}{|l|}{ Follow-up: } \\
\hline Died/not local/too frail & $5(9.6 \%)$ & $6(21.4 \%)$ & 0.21 & $0.44(0.11-1.62)$ \\
\hline No resp follow-up & $9(17.3 \%)$ & $2(7.1 \%)$ & 0.31 & $2.72(0.54-12.59)$ \\
\hline Resp follow-up $<3$ months & $37(71.1 \%)$ & $20(71.4 \%)$ & 1.00 & $0.99(0.36-2.73)$ \\
\hline Chest clinic review (days) & 52.5 (se 7.56$)$ & $36.7(\mathrm{se} 14.4)$ & 0.36 & n/a \\
\hline \multicolumn{5}{|l|}{ Advice: } \\
\hline Died & $3(5.8 \%)$ & $4(14.3 \%)$ & 0.23 & $0.36(0.08-1.77)$ \\
\hline No advice (of those alive) & $32(65.3 \%)$ & $13(54.2 \%)$ & 0.44 & $1.59(0.59-4.31)$ \\
\hline
\end{tabular}

and chest drain insertions were carried out in $A \& E$, reflecting the high proportion of A\&E referrals $-55.8 \%$ of the initial audit chest procedures, and an even higher proportion (71.4\%) in the re-audit. However, despite the high number of procedures performed in A\&E, 67.3\% (initial audit) and 66.7\% (re-audit) of the doctors performing the procedures were from the medical directorate and would be expected to have attended the educational update; this confirms that the two doctor groups were matched (OR 1.03, (0.38 - 2.77), $p=1.00$, data not shown) for doctor specialty and that a significant proportion did attend the intervention. There was a significant reduction in failure to record aspiration volume (41\% v 0\%, OR $15.4(0.026$ - 1.23), $p=0.04)$, and improvement of recording insertion in the "safe zone" (40.4\% v 84.6\%, OR $0.14(0.028-0.75), p=0.01)$. The chest drain gauge used was about 20 in both audits. Disappointingly, a significant proportion of cases failed to record either drain gauge (34.9\%) or dose of anaesthetic (55.8\%) with no significant improvement in the re-audit.

The operator grades are summarised in Table 4. There were no major differences between the two audits, with a predominance of Senior House Officer (SHO) operators. Table 4 also summarises the specialty referral response times. There was a significant drop in referral to the respiratory service in the re-audit (9.6\% v 28.6\%, OR 0.27 (0.07 - 0.91), p=0.05). Most respiratory referrals were seen well within the recommended 48 hours. Only a minority of referrals to thoracic surgery were reviewed after 72 hours despite there being no thoracic surgery service on site.

The follow-up arrangements and smoking advice offered on discharge are also summarised in Table 4. Non-significant improvements in both of these criteria were noted in the reaudit. In the initial audit, $17.3 \%$ of patients were not followedup in the chest clinic, and this improved to $7.1 \%$ in the re-audit. The average follow-up time improved from 52.5 days to 36.7 days. $65.3 \%$ of patients failed to receive smoking advice initially, and this figure improved to $54.2 \%$ in the re-audit.

The mortality rate was non-significantly higher in the reaudit (14.3\% versus $5.3 \%$ ). All cases were of secondary pneumothorax in an elderly group of patients (mean age 71.6 years) with limited underlying respiratory reserve. The 
underlying lung diseases were as follows: severe COPD (4 cases), severe pulmonary fibrosis (1 case), Panton Valentine Leucocidin staphylococcal pneumonia (1 case) and opportunistic tuberculosis infection (1 case). Two cases were transferred to intensive care and four cases were not deemed appropriate for intensive care due to comorbidity. In all cases the primary cause of death was sepsis - related either to the underlying disease or of nosocomial origin, but not to chest instrumentation or the pneumothorax itself.

\section{Discussion}

In this study, we compared SP management with that recommended in the 2003 BTS guidelines in an initial retrospective audit, and then re-audited management after an educational intervention. We detected significant management deviations - even when using a reduced $90 \%$ adherence standard - from the BTS algorithm, both in the initial audit and re-audit, with no overall improvement following the intervention.

\section{Management deviations}

The management deviations in this study were of four types: "conservative" (under-treatment of symptomatic SSP), "invasive" (over-treatment of symptomatic PSP), "incomplete" (failure to use oxygen with observation) and "inexplicable" (failure to insert a chest drain after unsuccessful aspiration).

The "conservative" strategy may result in failed resolution which may be serious and may lead to increased morbidity from more procedures and increased hospital stay. The "invasive" strategy could lead to increased morbidity related to unnecessary chest drain insertion and increased hospital stay. The "inexplicable" strategy is most serious as it could be life-threatening in chronic lung disease or in primary SP disease with a profound leak.

Several factors might explain such deviations. These include a lack of awareness of, or access to, guideline recommendations, despite the educational intervention. Some doctors may be unfamiliar with both pleural techniques. Doctors may perceive aspiration to be futile in a large PSP and may fail to appreciate the dangers of aspiration in SSP. The move to simpler Seldinger type drains may have reduced doctors' threshold to insert them. Or, some doctors may simply choose not to follow the guidelines.

The failure to proceed to chest drain insertion after unsuccessful aspiration is difficult to explain. Factors might include poor communication resulting from inadequate handover of patients from one shift to another, frequent shift changes, or lack of on-site expertise - even though Seldinger drains are technically easier to insert than Argyle drains.

\section{Effects of the intervention}

There was improved assessment and recording of pneumothorax size in the re-audit. However, this did not translate into improved management. This requires that the attending clinician must appreciate pneumothorax symptoms and identify any underlying lung disease which may have been underestimated by lack of spirometry and annotation of patients' symptoms.

There was a noted decrease in the rate of specialist referral in the re-audit. This may have been due to the greater proportion of PSPs in the re-audit cohort and greater confidence in managing pneumothoraces by non-respiratory specialties.

Recording of local anaesthetic doses was worse in the reaudit. Documentation and awareness is particularly important in order to minimise systemic toxicity by staying within the recommended doses $(<3 \mathrm{mg} / \mathrm{kg}) .{ }^{5}$ The average drain size used was 20Fr despite BTS guideline comments that large drains are only indicated for large leaks. ${ }^{3}$

A significant proportion of patients failed to receive chest clinic follow-up. This is important for a number of reasons: firstly, to confirm clinical recovery; secondly, to demonstrate chest $\mathrm{x}$-ray resolution of the pneumothorax which is required, for example, before air travel (although a six-week period for resolution is no longer required in the updated BTS 2004 air travel guidelines ${ }^{7}$ ); thirdly, to prevent recurrence; and finally, to detect possible underlying lung disease which may increase the possibility of future SP. It is also important that these details are communicated to GPs, to enable correct disease coding and so that advice can be given regarding management of future episodes, preventive strategies, and also since patients often consult their GP at the last minute regarding suitability for flying. According to the audit data, over $50 \%$ of patients failed to receive smoking advice, although it is not possible to determine whether or not this was due to poor record keeping.

The overall mortality rate in the combined audits (7 out of 80 cases, $8.8 \%$ ) is much higher than the disease-specific mortality noted in epidemiological studies. ${ }^{1}$ However, this is because the reasons for death were sepsis-related. It is notable that all cases were of secondary pneumothorax and had significant underlying disease in an elderly group of patients. The mortality data therefore do not reflect pneumothorax-specific mortality but are a reflection of the general physiological disturbance of the co-morbidities and underlying illnesses.

We have identified several failures in record keeping including documentation of CXR findings, site of drain insertion, dose of anaesthesia, chest drain gauge, and the provision of smoking advice.

\section{Previous studies}

Poor compliance with BTS pneumothorax guidelines is not new. Soulsby recorded a higher rate of aspiration (37\%) in a survey of patients with spontaneous pneumothorax attending a Liverpool A\&E department during 1993-98, although overall only about $20 \%$ of episodes were treated correctly according 
to the 1993 BTS guidelines. ${ }^{8}$ Mendis et al. highlighted poor familiarity with the 1993 BTS guidelines despite their study being conducted in a large London teaching hospital 2-5 years after the guidelines were available at the time. ${ }^{9}$ They found significant use of clamping and purse-string sutures. ${ }^{9}$ Yeoh et al. reported a lack of confidence in aspiration as a treatment for SSP or large PSP in a postal survey of consultant physicians and A\&E consultants in Wales. ${ }^{10}$

\section{Limitations}

There are several limitations to this study. The most important limitation arises from the different methodologies used. The initial audit was retrospective and subject to selection, coding and recall bias whereas the re-audit was prospective. Nevertheless, both cohorts were matched for age, gender and directorate with only five exclusions (only $6.3 \%$ of the total cases) in total because of failure to locate the records or coding error. In view of this, and the rigorous manner in which both audits were performed, we feel that the use of retrospective and prospective methods does not influence or preclude important conclusions on the efficacy of our intervention. We would also suggest that exclusions are an inevitable feature of most such studies.

Another key limitation was that the initial target audience did not include the A\&E staff although they would have had similar Trust intranet access to the updated guidelines. Despite this, $66.7 \%$ of doctors in the post-intervention group had attended the education update - which is almost identical to the $67.3 \%$ of doctors from the same directorate in the original retrospective audit. Therefore, the proportion of doctors from the same directorate was matched. However, as only $66.7 \%$ of doctors in the post-intervention group attended the education update, future studies are required to compare the relative performance of attendees and non-attendees of the update in order to define more closely the true efficacy of the intervention. Nevertheless, we feel that the exposure of a significant majority to the intervention allows conclusions to be drawn on its efficacy in the interim.

The patient numbers were limited by incidence and coding factors, thereby limiting statistical power, but the case numbers do allow meaningful statistical comparison. The cohorts were not matched for proportions of PSP and SSP cases, which may explain the reduced use of chest drain and increased use of 'observation only' in the re-audit cohort where the proportion of PSP cases was higher.

Pneumothorax size was assessed from the digital CXR image, not a wet film with a tendency to technical underestimation. Conclusions were based on the medical records. Correct (or incorrect) practices that were not documented would therefore be undetected. Underlying lung disease was probably underestimated due to a lack of spirometry data in patients with a significant smoking history.

Chest drain complication rates were not formally audited since drain observation charts were not used, filed or obtainable, although no complications of pain, misplacement or dislodgement were noted in the records. This again may reflect failure to record data, but Seldinger type drains are perceived as being safer to insert. This impression is supported by a recent prospective observational study which confirmed a very low complication rate of less than $2 \%$, comparing favourably with historical controls. ${ }^{11}$

Several other points should be noted. Hospital bed stay data were not available. Also, installing the Trust intranet guideline hyperlink involved input from IT departments, thereby introducing inevitable delay. And finally, at the time of this study, the UK junior doctor foundation programme was still in its early stages and 'F2' doctors were not in post.

\section{Potential strategies}

The failure of the educational intervention is concerning and cannot wholly be accounted for by methodological or training attendance factors. Of course, it is important to calculate the true efficacy of the educational intervention by comparing the performance of attendees and non-attendees. We suggest that a pneumothorax proforma containing key data from the BTS guidelines might be a simple tool to improve the record keeping failures we have noted. The management deviations are more concerning and might be improved by development and use of a pheumothorax care pathway containing the BTS 2003 algorithm. In addition, it is important to target all directorates' junior (and senior) staff with any future educational intervention and repeat it at intervals consistent with the rotation of junior staff - i.e. 4-6 monthly. Use of IT to consolidate the key message is useful but should be implemented speedily by minimising the number of interventionalists in the loop and reducing bureaucracy. Future prospective re-audits using BTS criteria are required to assess any change in quality of SP management in response to these interventions.

\section{Conclusion}

This study has identified several areas where current management of spontaneous pneumothorax deviates from the revised 2003 BTS guidelines. There is a precedent for such deviations. There was no overall improvement in management following an educational intervention, but not all doctors in the post-intervention group attended the education update; a further study comparing the performance between attendees and non-attendees is warranted in order to assess the true efficacy of the intervention. The number of randomised controlled trials in SP management is still small and it is likely that revised BTS guidelines will be needed in the future to complement advances in research and understanding. Guidelines are now more easily accessible online. The challenge is how to modify practice so that it follows guideline recommendations. Suggestions include a pneumothorax 


\section{Box 1. Key Learning Points.}

1. Adherence to the updated BTS pneumothorax guidelines in a district general hospital is poor despite initial educational interventions.

2. The greatest concerns are failure to insert a chest drain after failed aspiration and attempting to aspirate symptomatic SSPs.

3. Other unexpected findings were the magnitude of record keeping errors and high proportion of procedures performed in A\&E.

4. Identification of the complete target audience and reducing the number of personnel involved to effect change is essential.

5. A further study comparing the relative performance of intervention attendees versus non-attendees will define more closely the true efficacy of the intervention.

6. Future strategies to improve standards might include a pneumothorax proforma to improve aspects of record keeping, and a pneumothorax care pathway to reduce management errors.

proforma to improve record keeping, and a pneumothorax care pathway to reduce management deviations. Other prerequisites involve educating the complete target audience at the outset and at regular intervals, and minimising the number of personnel required to implement the intervention process. The Key Learning Points from this study are shown in Box 1.

\section{Acknow ledgements}

We thank staff of the Clinical Audit and Medical Records Departments at Musgrove Park Hospital, Taunton.

\section{Conflict of Interests}

None.

\section{Funding}

None.

\section{References}

1 Gupta D, Hansell A, Nichols T, Doung T, Ayres JG, Strachan D. Epidemiology of pneumothorax in England. Thorax 2000;55:666-71.

2 Miller AC, Harvey JE. Guidelines for the management of spontaneous pneumothorax. BMJ 1993;307:114-16

3 Henry M, Arnold T, Harvey J, Pleural Disease Group, Standards of Care Committee, British Thoracic Society. BTS guidelines for the management of spontaneous pneumothorax. Thorax 2003;58(Suppl ii):ii39-ii52.

4 Light RW. Pneumothorax. In: Pleural Diseases. 3rd edition. Baltimore: Williams and Wilkins, 1995: 242-77.

5 Laws D, Neville E, Duffy J, Pleural Disease Group, Standards of Care Committee, British Thoracic Society. BTS guidelines for insertion of a chest drain. Thorax 2003;58(Suppl ii):ii53-ii59.

6 Baumann MH, Strange C, Heffner JE, et al.; AACP Pneumothorax Consensus Group. AACP Pneumothorax Consensus Group. Management of spontaneous pneumothorax: an American College of Chest Physicians Delphi consensus statement. Chest 2001;119(2):590-602

7 BTS Air Travel Working Party. Managing passengers with respiratory disease planning air travel. BTS recommendations, BTS Standards of Care Committee. Online: http://www.brit-thoracic.org.uk/c2/uploads/FlightRevision04.pdf.

8 Soulsby T. British Thoracic Society guidelines for the management of spontaneous pneumothorax: do we comply with them and do they work? J Accid Emerg Med 1998;15:317-21.

9 Mendis D, El-Shanawany T, Mathur A, Redington AE. Management of spontaneous pneumothorax: are British Thoracic Society guidelines being followed? Postgrad Med J 2002;87:80-4.

10 Yeoh JH, Ansari S, Campbell IA. Management of spontaneous pneumothorax - a Welsh survey. Postgrad M ed J 2000;76:496-500.

11 Horsley A, Jones L, White J, Henry M. Efficacy and complications of small-bore, wire-guided chest drains. Chest 2006;130(6):1857-63.

\section{Available online at http://w w w.thepcrj.org}

